\title{
A new species of Sarcocystis in the brain of two exotic birds
}

\author{
by P. C. C. GARNHAM, A. J. DUGGAN and R. E. SINDEN *
}

Imperial College Field Station, Ashurst Lodge, Ascot, Berkshire

and Wellcome Museum of Medical Science, 183 Euston Road, London N.W.1., England.

\section{Summary.}

Sarcocystis kirmsei sp. nov. is described from the brain of two tropical birds, from Thailand and Panama. Its distinction from Frenkelia is considered in some detail.

\section{Résumé.}

Une espèce nouvelle de Sarcocystis dans le cerveau de deux Oiseaux exotiques.

Sarcocystis kirmsei est décrit du cerveau de deux Oiseaux tropicaux de Thaillande et de Panama.

Les critères de distinction entre cette espèce et le genre Frenkelia sont discutés en détail.

In 1968, Kirmse (pers. comm.) found a curious parasite in sections of the brain of an unidentified bird which he had been given in Panama. He sent unstained sections to one of us (PCCG) and on examination the parasite was thought to belong to the Toxoplasmatea, either to a species of Sarcocystis or of Frenkelia. A brief description of the infection was made by Tadros (1970) in her thesis for the Ph. D. (London). The slenderness of the cystozoites resembled those of Frenkelia, but the prominent spines on the cyst wall were more like those of Sarcocystis. The distribution of the cystozoites within the cyst is characteristic in that the central portion is practically empty while the outer part consists of numerous pockets of organisms, closely packed together. The parasite could not be named in the absence of a specific identification of the host.

However, eight years later, during the re-arrangement of Wenyon's collection of protozoa, one of us (AJD) encountered a strange parasite in a section of the brain

Accepté le 23 février 1979. 
of a bird which had died in the gardens of the Zoological Society of London in 1936. On examination, the parasite was found to bear a striking resemblance to the Panama infection. Fortunately, full details of the second example are available. The bird was a male Siamese Fire-Backed Pheasant (Lophura diardi) which had been acquired by the Zoo on 21st March, 1934. It died two years later (21st February 1936) and an autopsy was performed the same day. Signs of old age, emaciation and anaemia were present; the kidneys were enlarged and oedematous, and chronic interstitial nephritis was thought to have been the primary cause of death. The brain was noted as being very anaemic with? softening; sections were prepared and the pathologist (Dr. A.E. Hargreaves) found cysts which he thought contained numerous trypanosomes. This identification was incorrect, as they were undoubtedly the slender elongated cystozoites of a coccidian parasite.

Although blocks of brain or other organs of the second example were missing, several slides had been preserved, on which we base a description of the parasite. The coverslip over two sections was removed and the tissue was prepared for electron microscopy. Although the ultrastructure was of the poorest quality, it revealed a few points of interest.

\section{Morphology of cysts in brain of $L$. diardi}

Three or four cysts were found in each section (in the original haematoxylin and eosin staining). They were present in the grey and white matter of the cerebrum.

The cysts were spherical (fig. 1) and without the lobulation characteristic of some species of Frenkelia (e.g. F. microti). However the presence of apparently two cysts in close proximity was suggestive that they might have been joined in the form of a single, bilobed parasite. The diameter varied from 750-1 $100 \mu \mathrm{m}$. The massed cystozoites were grouped into packets (fig. 2), not unlike the cytomeres of a megaloschizont of Leucocytozoon except that the contents were long cystozoites instead of tiny merozoites. It was sometimes difficult to distinguish a wall around the compartments which lay in close apposition and yet kept their individuality.

The cystozoites were elongate, banana-shaped bodies $8-9 \mu \mathrm{m}$ in length with one end more pointed that the other. The nucleus was single and usually centrally placed; a prominent vacuole was often present (fig. 3).

An important character was the structure of the cyst wall. The width was about $4 \mu \mathrm{m}$; projecting outwards were spines, readily seen under the light microscope (fig. 4), and rising perpendicularly about $3 \mu \mathrm{m}$ from a wide base to the points, about $4 \mu \mathrm{m}$ apart. The inner portion of the cyst wall was amorphous, except that it occasionally appeared to have vertical striations or bands corresponding to spines which the folding of the section had apparently displaced. The cyst wall extended into the interior as septa and provided the material for the envelope of the oval corpuscules. This envelope was thickened, about $2 \mu \mathrm{m}$ wide and was best seen around the empty bodies. 


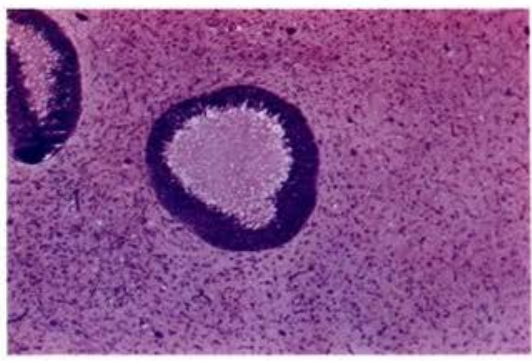

Cyst in cerebrum of Thai bird. $\times 30$

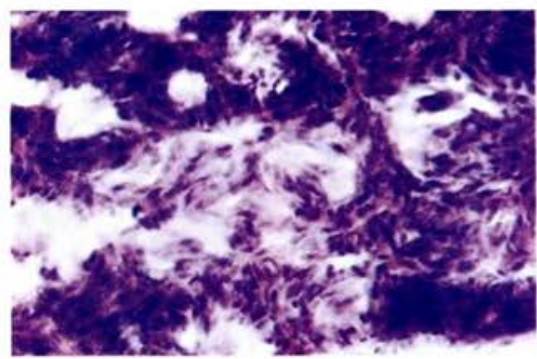

Individual cystozoites. Thai bird. $x 1950$

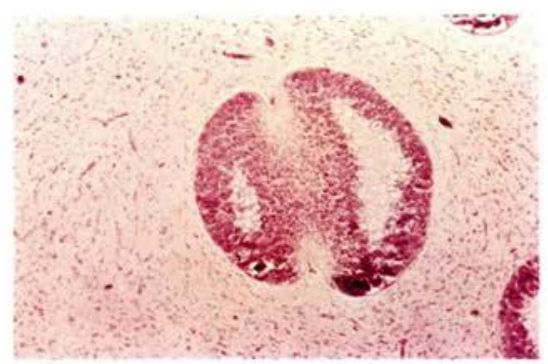

Cyst in cerebrum of Panama bird $\times 30$

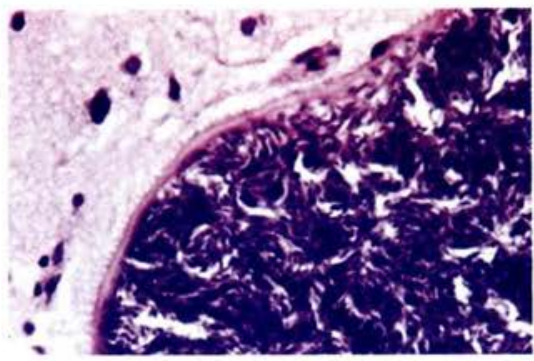

Packets of cystozoites. Thai bird. $\times 530$
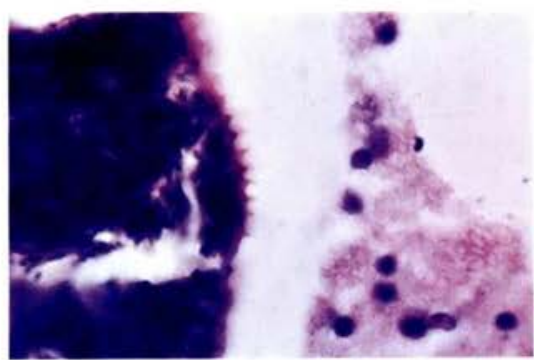

Spines of cyst wall from Thai bird. $\times 1950$

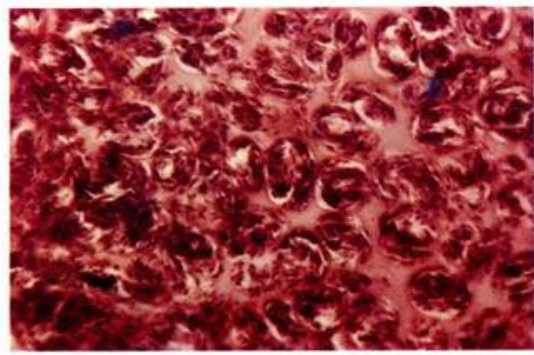

Packets of cystozoites. Panama bird. $\times 400$

Planche I. A New Species of Sarcocystis in the Brain of Two Exotic Birds

Figures 1 to 4 are taken from sections of brain of the Thailand bird. Figures 5 and 6 are taken from the brain of the Panama bird.

1) Cyst in cerebrum. $\times$ 30. (H. and E.). 2) Packets of cystozoites. $\times 450$. ( H. and E.). 3) Cystozoites. $\times 750$. 4) Cyst wall showing spines. $\times 750$. 5) Cyst in cerebrum. $\times$ 30. (H. and E.). 6) Packets of cystozoites. $\times 300$. (H. and E.).

Note. - Les grossissements indiqués sur la figure sont erronés. Ils n'ont pu être modifiés pour des raisons techniques. Mais ils sont exacts dans la légende. 
The ultrastructure is of little help in generic differentiation, owing to loss of detail in the unsuitably fixed material of ancient provenance. The cyst wall ( $f i g .7$ ) showed projecting spines of an apparently simple structure and of dimensions about the same as those visible under light microscopy (height 1.5-2 $\mu \mathrm{m}$ ). Longitudinal strands along the spines (fig. 8) might represent a highly disorganised fibrous sheath, or microtubules which occur in some species of Sarcocystis. Polar rings (fig. 9) in transverse and tangential section were visible and had a diameter of $23-42 \mathrm{~nm}$. A few sections of cystozoites revealed cystozoites filled with numerous micronemes with a diameter of $70 \mathrm{~nm}$ (fig. 10).

\section{Morphology of cysts in brain of Panama bird}

Many cysts (fig. 5) were found in sections of the cerebrum and cerebellum, stained with Giemsa, Masson, Silver and periodic acid-Schiff (P.A.S.).

Kirmse (unpublished) gives the following description of the parasite, the dimensions corresponding closely with our own from the same material (kindly supplied by Dr. P. Kirmse). The cysts were oval or spherical and occasionally bean-shaped, and measured from $75 \mu \mathrm{m}-450 \mu \mathrm{m}$. The contents comprised chambers (fig. 6) packed with cystozoites and irregularly divided by trabeculae dipping inwards from the cyst wall; the central portion contained almost empty chambers of variable size (from 3-16 $\mu \mathrm{m})$. The cyst wall had a double membrane from the surface of which radial spines emerged at regular intervals of $2 \mu \mathrm{m}$. The cystozoites were crescentic, usually with one end pointed and the other blunt and measured between eight and $9 \mu \mathrm{m}$ in length and about $2 \mu \mathrm{m}$ in width. The cyst wall was argyrophilic and P.A.S. negative; the cystozoites were strongly P.A.S. positive.

Our measurements of the dentate projections gave a length of $2.5 \mu \mathrm{m}$ and a space of $2.5-3 \mu \mathrm{m}$ between each. The cyst wall was about $3 \mu \mathrm{m}$ in width, the cystozoite band about $100 \mu \mathrm{m}$ and the empty core $200 \mu \mathrm{m}$.

Except for the overall size, the structure of the cyst was precisely the same as that of the Asian parasite, i.e. an almost empty core save for the remnants of chamber walls and a few stray cystozoites, and surrounded by a wide band of massed packets of cystozoites.

\section{Differential diagnosis}

Coccidial cysts have been found in the brains of many mammals suffering from toxoplasmosis but less often in birds. The brain cysts of Toxoplasma gondii are readily identified by 1) limitation of size to a maximum of $150 \mu \mathrm{m}, 2$ ) the thin cyst wall (with cog-like projections visible in electron microscopy), 3) the spherical outline, 4) the size and uniformity of the cystozoites (2 to $5 \mu \mathrm{m})$ and 5) the strongly positive P.A.S. reaction. The allied genus, Hammondia, of dubious validity, is not discussed here. 


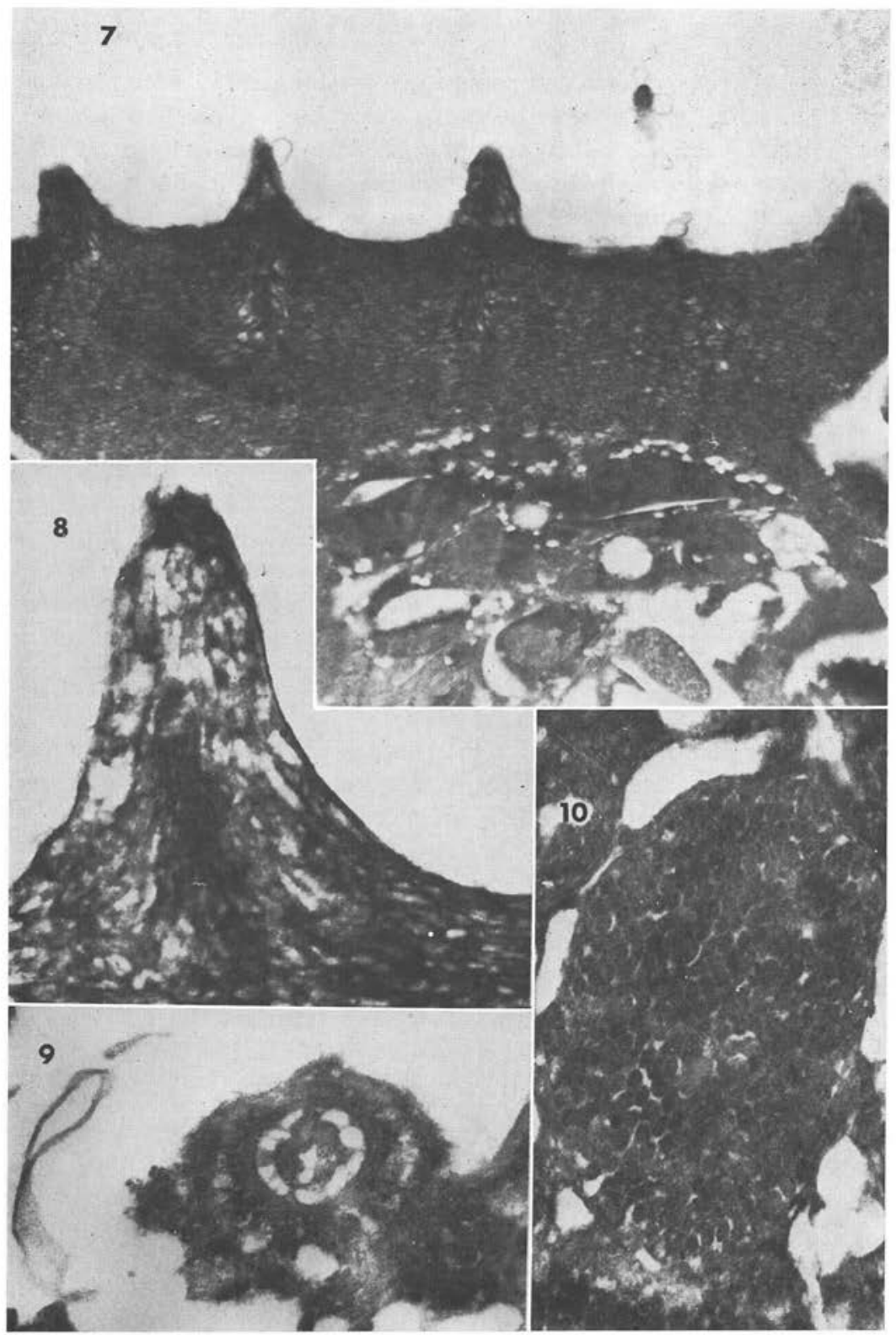

Planche II. A New Species of Sarcocystis in the Brain of Two Exotic Birds

Electronmicrographs of the cyst from the Thailand bird.

7) Cyst wall showing spines. $\times 9,500 .(\mathrm{EM}) .8$ ) Cyst wall spines. $\times 31,000 . \quad(\mathrm{EM})$. 9) Polar rings in cystozoite. $\times 69,000$. (EM). 10) Micronemes in cystozoite. $\times 46,000$ (EM). 
The cysts of Frenkelia spp. are confined to the brain and so far have only been found in a limited range of cricetid rodents. They have a characteristic morphology: 1) the size varies greatly, but they are often visible to the naked eye, 2) the outline is either lobulate or spherical, 3) the cyst wall is relatively thin, but thicker than that of Toxoplasma; the ultrastructure reveals three portions; an outer " toothed » wall, a thicker inner layer of amorphous character which dips into the interior at intervals and a space of varying size in the centre, 4) the cystozoites are thin, about 7-9 $\mu \mathrm{m}$ in length and P.A.S.-positive.

The cysts of Sarcocystis were once thought to be confined to muscle, but in a few instances, they have been found in the brain of mammals, chiefly sheep and cattle. Tadros and Laarman (1978) have summarised the findings, and have reported the largely negative results of their own observations on the brain of cattle and rodents. In the former they were only able to detect scanty cystozoites after pepsin digestion; in the latter, they found numerous cysts in the brains of two experimentally infected Apodemus sylvaticus. In the earlier records, the cerebral sarcocysts measured from 34-143 $\mu \mathrm{m}$ in diameter, had radially striped smooth cyst walls and a septate interior with round or sausage-shaped cystozoites. The septate interior, the length (up to $15 \mu \mathrm{m}$ ) and shape of the cystozoites clearly indicate the generic position of the parasite.

The problem here is to determine the genus. The three possibilities are Sarcocystis, Frenkelia or a new genus. The paucity of material does not warrant the creation of a new genus. Sarcocystis is quite common in the muscle of many birds; Levine (1978) lists 30 avian hosts while Kalyakin and Zasukhin (1975) record 70 species; in both lists, water birds are the most frequent hosts. However cerebral invasion was not reported in any of these instances. On the other hand, Frenkelia in its intermediate host appears to be limited to the brain and only to rodents. It is difficult to explain the presence of a single species in the brain of two birds - one a pheasant, the other a passeriform, in places as far apart as Thailand and Panama. Outbreaks of Sarcosporidiosis have been reported by Munday et al. (1971) in domestic fowls in Papua but no cerebral involvement was noted.

Morphologically, the parasites under discussion resemble Frenkelia in the slenderness of the cystozoites and the fairly thin cyst wall. The "empty " or degenerating interior is seen equally in species of Frenkelia and Sarcocystis. The spines on the surface of the cyst are more like those described in certain species of Sarcocystis, rather than the cauliflower-like projections present in Frenkelia. Mehlhorn et al. (1976) describe and illustrate the cyst wall of a species of Sarcocystis found in domestic hens in New Zealand; palisade-like protrusions with a length of $1.5 \mu \mathrm{m}$ were present and although not pointed like the ones under discussion, there is a general similarity; the spacing between the spines is rather less - under $2 \mu \mathrm{m}$ instead of $2 \mu \mathrm{m}$ or over. The presence or absence of metrocytes does not provide a certain distinction for they have been seen in both genera (Tadros, 1970); moreover in the present material they were not identified with certainty. Frenkel (1977) 
discusses the identification of the coccidian parasites found in the tissues of the intermediate host, and in the intestine of their definitive hosts; the details of the respective life cycles of Sarcocystis spp. and Frenkelia spp. are practically the same, while there is little difference in the morphology of the tissue cysts. In the intermediate host the host cell nucleus is said to be enlarged in Frenkelia, but in natural infections of the latter, nuclei are usually invisible.

Frenkelia spp. are relatively localised parasites both geographically and in regard to f.osts, unlike the cosmopolitan Sarcocystis, while Frenkelia appears to be restricted to rodents in temperate zones; both examples of the present material come from the tr pics. The ultrastructural criteria of distinction are minimal.

In view of the above considerations, we place this organism in the genus Sarcoystis and rame it Sarcocystis kirmsei in honour of Dr. Peter Kirmse who first saw the parasite 11 c... unidentified bird in Panama. The material formed part of a pool of four birds as follows:

Rhyncocyclus brevirostris (fam. Tyrranidae),

Stelgidopteryx ruficollis (fam. Hirundinidae),

Myioborus miniatus (fam. Parulidae),

Spinus xanthogaste: (fam. Fringillidae).

Except for the larger size of the cysts in the pheasant, the appearance of the two examples of this parasite is almost identical, and is remarkable in view of the geographical separation of the two localities. This group of coccidial parasites is likely to be very primitive and its characters have become firmly fixed.

\section{Diagnosis}

NAME: Sarcocystis kirmsei sp. nov.

VERTEBrate host: Lophura diardi (family Phasianidae).

Locality: Thailand (London Zoological Gardens).

CHARActers: Forms subspherical cysts up to $1,100 \mu \mathrm{m}$ in size in the brain. The cyst wall has characteristic spines and the contents consist of an almost empty central portion and a wide outer portion containing thousands of slender cystozoites arranged in oval packets.

OTHER Hosts: An unidentified passerine bird in Panama (Cerro Punta, Chiriqui Province, at an altitude of $1,700 \mathrm{~m}$ ).

TyPE MATERIAL: A section of the brain of the Thai bird (W1003) and of the Panama bird (W1003A) are held at the Wellcome Museum of Medical Science. Sections of the brain of the Thai bird are also held at the London Zoological Gardens (195/36). 


\section{References}

Frenkel J.K. (1977): Besnoitia wallacei of cats and rodents with a reclassification of other cystforming coccidia. J. Parasitol., 63, 611-628.

Kalyakin V.N., Zasukhin D.N. (1975): Distribution of Sarcocystis (Protozoa: Sporozoa) in vertebrates. Folia Parasitol. (Praha), 22, 289-307.

Kirmse P. (Personal Communication): Description of a Sarcocystis-like organism in the brain of a bird from Panama.

Levine N. D. (1978): Species of Sarcocystis. (In press).

Munday B. L., Humphrey J.D., and Kila V. (1977) : Pathology produced by, prevalence of, and probable life-cycle of a species of Sarcocystis in the domestic fowl. Avian Dis., 4, 698-703.

Tadros W. A. (1970): Studies on the M-organism and related protozoan parasites of rodents. Ph. D. thesis, University of London.

Tadros W. A., Laarman J. J. (1978): Observations on cerebral sarcosporidiosis: natural incidence in cattle and experimental infection in the rodent Apodemus sylvaticus, together with a simple guide for differential diagnosis of cysts of related eimeriid coccidia occurring in the nervous system of vertebrates. (In press). 